\title{
El trabajo social de empresa: otro ámbito de trabajo
}

\author{
Fayna Mesa Medina ${ }^{1}$ \\ Recibido: 08-05-2015 - Aceptado: 13-06-2015
}

\begin{abstract}
Resumen
Cuando se habla de Trabajo Social se relaciona únicamente con servicios sociales (ssss), desde el enfoque de solucionar los problemas de la sociedad, sin embargo, la función del profesional de Trabajo Social (TS) es acompañar a las personas en los momentos de cambio, fomentando y contribuyendo al desarrollo de Bienestar Social. El Trabajo Social de Empresa (TSE) es otra de las áreas de la profesión de Trabajo Social, aún por desarrollar, dado que en las Universidades la formación que se imparte se vincula principalmente a los servicios sociales. La hipótesis de la que parte este estudio considera que la figura del trabajador social tiene un lugar relevante en el departamento de Recursos Humanos en las empresas, donde puede desarrollar su ejercicio como profesional experto en el ámbito social, dedicándose al bienestar ocupacional. Este principio plantea como objetivo general dar visibilidad a la figura del TSE, favoreciendo que profesionales de Trabajo Social conozcan la figura mencionada. Como objetivos específicos, se establecen averiguar el papel que ejerce el TSE, cuál es su situación e investigar cómo ejercen su función. Esta investigación tiene una metodología cualitativa, de carácter descriptivo y aplicado, donde se utiliza la técnica de la entrevista en profundidad; específicamente entrevista enfocada. Los resultados obtenidos en el estudio indican que el profesional de TSE promueve el bienestar laboral; previniendo conflictos laborales, informando y orientando sobre los recursos que favorecen la conciliación familiar, ofreciendo soporte a los cambios personales que se producen de la fase productiva a la jubilación. Como conclusión, se refleja una mejora de las condiciones de trabajo cuando la empresa dota al departamento de recursos humanos de un profesional de TSE.
\end{abstract}

Palabras clave: recursos humanos, trabajo social de empresa (TSE), bienestar ocupacional.

\section{Company social work: another field of work}

\begin{abstract}
When we are referring to Social Work, it seems that it's only related to social services, from the standpoint of solving the problems of society. However, the Social Worker's role is to accompany people in times of change, promoting and contributing to the development of their Social Welfare. Therefore, the Social Work Company (SWC) is another area of the Social Work profession, still in process of development, as the
\end{abstract}

${ }^{1}$ Magíster en Atención sociosanitaria a la dependencia. Magíster en Mediación Intercultural y participación ciudadana. Trabajadora social por la Universidad de Valencia. Trabajadora de Instituto Valenciano de Acción Social (IVAS). Valencia, España; fayna_mm@ hotmail.com 
training provided in universities is still mainly linked to social services. This research begins considering the hypothesis that the figure of the Social Worker has an important place in the HR department in companies, where it can develop its exercise as a professional expert on the social field, devoted to Occupational Welfare. This principle has as its general objective to give visibility to the figure of the SWC, favoring that Social Work professionals and students know the figure mentioned. The specific objectives are to determine the role played by the SWC, what their situation is and investigate how they exert their function. This research has a qualitative methodology, descriptive and applied nature, where the technique of in-depth interview is used; specifically focused interview. The results of the study indicate that SWC professionals promote workplace wellness; preventing labor disputes, reporting and focusing on resources that promote life balance, offering support to personal changes that occur in the production phase to retirement. In conclusion after analyzing the results, the working conditions are improved when the company gives the human resources department, a SWC professional.

Keywords: human resources, social work company (SWC), occupational welfare.

\section{Introducción}

El pensamiento empresarial ha tenido una gran evolución a lo largo de la historia, la empresa era concebida, exclusivamente, como una unidad económica cuya finalidad era obtener beneficios.

El papel del trabajador social de empresa se articula en torno a tres planos de actuación dentro de la empresa, empezando por aportar una ayuda psico-social a los individuos y a las colectividades de trabajadores que se encuentran en dificultades; participar en la creación, en el funcionamiento y en la mejora de las realizaciones sociales de la empresa y favorecer una mejor toma de conciencia de los problemas humanos y sociales surgidos con motivo del trabajo.

Sin embargo, Carrasco (2009) apunta que esta forma específica del Trabajo Social de Empresa entró en declive de forma progresiva, pudiéndose establecer como detonante las dificultades de identidades del trabajador social de empresa, el desarrollo y consolidación del estado de bienestar y las transformaciones en el mundo empresarial.

Nacimiento y evolución del Trabajo Social de Empresa

Para situarnos en el tiempo es necesario retroceder hasta antes de la Revolución Industrial, la mayor parte de las organizaciones eran pequeñas, y el trabajo lo realizaban muy pocas personas, pero a partir de la Revolución Industrial, se empieza a producir la división en el trabajo asumiendo a la persona como "una máquina más", de tal forma que se consiguió multiplicar la productividad, objetivo primordial y promoviendo el surgimiento de grandes empresas.

El Trabajo Social de Empresa surge en los países industrializados más avanzados del siglo XIX como son Inglaterra, Bélgica, EE.UU., apareciendo como mecanismo de mediación entre el capital y el trabajo. Pero, no hay que obviar que una empresa es también, un sistema donde interactúan los elementos que la componen, en el que el activo más importante son las personas que trabajan en ella.

Es en ese momento cuando se inicia el compromiso de las empresas de hacerse cargo de corregir los rigores del capitalismo, proteger el sistema de una posible revolución y mantener en buen estado la fuerza del trabajador (Castillo, 2010).

Por ello, durante el siglo XX, especialmente tras la II Guerra Mundial, se pasa de un modelo taylorista de la empresa, carente de horizontes sociales y ambientales y centrado en el rendimiento económico, a un modelo más cohesionado internamente y más comprometido con el entorno (García Perdiguero, 2003, citado en Relinque y Burgos, 2008, p. 234). 
BÚSQUEDA - Enero / Junio de 2015 - No. 14 (62 - 76)

Para lograr que la empresa sea socialmente responsable, además de cumplir sus obligaciones legales vigentes, tiene que asumir, voluntariamente, en todos los rangos de cargos, políticas y procedimientos, las preocupaciones sociales, laborales, medioambientales y de respeto a los Derechos Humanos que surgen de la relación y el diálogo transparente con sus grupos de interés, comprometiéndose a las consecuencias e impactos que derivan de sus acciones (Carrasco, 2009).

Así, se reconoce que los trabajadores son algo más que un simple factor de la producción, son seres humanos que tienen que alcanzar la plenitud en todos los aspectos, y, por ello, es el trabajador social el profesional adecuado para facilitar este proceso, porque conoce y puede proporcionar los medios para lograr esta realización humana, en la parcela de trabajo que le concierne.

"Entendiendo de esta manera la gestión empresarial, nos encontramos con la circunstancia de que la profesión de Trabajo Social puede tener mucho que decir y hacer" (Carrasco, 2009, p.69).

Según la autora Rodríguez (2010), antes de 1960 la figura del trabajador social de empresa realizaba las funciones de intermediario entre los trabajadores y la dirección, era un reforzador del buen ambiente entre los elementos de producción y era considerado un "ente de bondad" que repartía beneficencia patronal con equidad y eficacia.

De este modo, se deduce que a través del Trabajo Social de Empresa se puede lograr el bienestar para los trabajadores, mediante un buen ambiente laboral que ahuyenta conflictos sociales, favoreciendo así una mayor productividad con el consiguiente rendimiento económico para la empresa (Soto, 1991, p.744).

Es Mercedes Marcina (1989), trabajadora social de Telefónica en Barcelona, quien realiza una sinopsis del pasado del Trabajador Social de Empresa en España diciendo que:
A partir de la década de los 60, comienza a surgir en España, la asistencia social como forma de prestación de servicios a los más necesitados. Ante la carencia de programas mínimos de Bienestar Social, este papel fue asumido por muchas empresas, obligadas por la Administración, de manera espontánea o a demanda de los trabajadores, por lo que comenzaron a implantar en sus centros de trabajo una serie de prestaciones sociales que, de forma paternalista, ofrecen a sus empleados. (Citado por Marcuello, 2006, p. 16).

Fue en 1963, cuando el grupo de Trabajadores Sociales de Barcelona pensaba que "la característica peculiar del Trabajo Social de Empresa es la de ayudar a resolver los problemas que se suscitan en relación con la situación de las personas en la empresa y el trabajo en la misma" (II Seminario de Trabajadores Sociales, 1989).

Es en este momento, cuando la profesión del Trabajo Social de Empresa comienza a verse como un profesional de importancia en las obras sociales dentro de una empresa, dirigiendo cada vez más su actuación hacia la participación en la elaboración y puesta en práctica de la política social de la empresa, buscando el desarrollo de las personas que en ella trabajan y el logro del bienestar ocupacional (Roldán, 1990).

En los años 80, y como resultado de la crisis que se vivió en los años 70, el Estado español deja de ser el único administrador del "gasto social" y el responsable de la contención de las desigualdades que se vivían en esa época. Comienza la idea que no solo se hará cargo el Estado, sino que también hacen responsable del bienestary de la calidad de vida a las instituciones sociales, a la empresa, ya sea lucrativa o no (De la Cuesta y Valor, 2003).

La consolidación de sistemas públicos en el ámbito de la sanidad, la educación y los servicios sociales se constituyó como la causa vertebral de la desaparición del Trabajo Social de Empresa. El Estado pasó a ser el garante de unos derechos ciudadanos que ya no tenían que resolverse en instancias privadas. 
Fayna Mesa Medina - El trabajo social de empresa: Otro ámbito de trabajo

De esta forma, en el momento que se consolidó un servicio público que atendía a las necesidades de carácter educativo, sanitario y/o social, las empresas dejaron de prestar un servicio que, para ellas, era secundario y prescindible.

"El desarrollo de un sistema social de carácter público y la transformación producida en el seno de las empresas redundaron en la desvirtuación del papel del trabajador social en este ámbito de intervención" (Carrasco, 2009, p.71).

\section{Definición de Trabajo Social de Empresa \\ El Trabajo Social Empresarial se define como: La actividad organizada que pretende ayudar a la adaptación recíproca de los trabajadores y su empresa. Este objetivo se alcanza mediante la utilización de técnicas y métodos destinados a permitir que los trabajadores, los grupos y las colectividades de trabajo, hagan frente a las necesidades, resuelvan los problemas que plantea su adaptación a una sociedad industrial en evolución y, merced a una acción cooperativa, que mejoren las condiciones económicas y sociales (Bernard, 1967, p.19).}

Se estudian y trabajan desde conductas adictivas, solicitudes de cambio de puesto en un departamento con numerosas peticiones hasta la mejora de la comunicación interna de la empresa, en el que se pueden marcar aspectos relevantes y que interrelacionan como aumentar la vinculación del trabajador con la empresa, aspecto que lleva asociado el aumento de la productividad y redundando en la obtención de un mayor beneficio para la propia entidad y porque es importante lograr un buen ambiente laboral para el buen desarrollo profesional de los trabajadores (Soto, 1991).

La definición elaborada por el Grupo de Trabajadores Sociales de Empresa del Colegio Profesional de Trabajo Social de Madrid permite aclarar sus objetivos y funciones:

Favorecer el bienestar de los trabajadores mediante la mejora de los aspectos laborales, sociales, sanitarios, personales y familiares, a través de las gestiones internas y externas oportunas para conseguir el equilibrio de los intereses de la empresa, para un dar un mejor servicio a la empresa y a la sociedad. (Raya y Caparrón, 2013, p.343).
La realidad de una empresa es totalmente compleja, en ella se dan problemas tecnológicos, psicológicos, sociales, económicos, pero quizá el aspecto humano es el más complejo debido a las propias necesidades estructurales, emocionales, intelectuales y culturales de la persona, motivos por los que el activo más importante que tiene una empresa es el de las personas que trabajan en ella (Gorostidi y Cebrián, 1990).

De hecho, tal y como afirma el autor Soto (1991): "La empresa modelo es aquella que más y mejor produce y la que proporciona mayores beneficios sociales a sus trabajadores" (p.2). Por ello, se considera como signo de calidad que el Trabajador Social esté integrado en la plantilla de la organización (Rodríguez, 2010).

Es un trabajo a largo plazo y es necesaria la estabilidad en el puesto para trabajar conjuntamente con el departamento de Relaciones Laborales. Además, para la consecución de los objetivos del Trabajador Social de Empresa, se requiere el conocimiento paulatino de la plantilla, de las políticas de la empresa y a su vez, el compromiso de su mejoramiento como parte integrante de la propia empresa.

No obstante, es relevante destacar que existen dificultades de identidad del Trabajo Social de Empresa, puesto que en mayo de 1985 en Madrid, en la sede de CAMPSA, acudieron al I Seminario de Trabajo Social de Empresa 142 asistentes sociales pertenecientes a 75 empresas en España. En Madrid, en 1992, había 57 Trabajadoras Sociales, pertenecientes a 32 Empresas (Rodríguez, 2010).

Del mismo modo, en el año 2010 los datos no son precisamente alentadores, de esas 32 empresas con sede en Madrid que existían y que contaban con el profesional de Trabajador Social, solo se tienen datos positivos de 14 , que siguen conservando el puesto en la empresa (Rodríguez, 2010).

Sin embargo, el Trabajo Social de Empresa privada constituye hoy en día una relevante alternativa al Trabajo Social en las Administraciones Públicas y ONG's, puesto que 
BÚSQUEDA - Enero / Junio de 2015 - No. 14 (62 - 76)

el Trabajo Social se define por su versatilidad e implicación en la realidad existente.

Esto establece las formas, demandas, aplicaciones y concreciones de la disciplina. Una de ellas es el Trabajo Social de Empresa Privada, llamado también Servicio Social de Empresa o Trabajo Social Ocupacional, que es un modo de aplicar los contenidos propios del Trabajo Social al mundo de las relaciones industriales, laborales o de la empresa.

"Si se quiere propagar el T.S. en la Empresa habrá que buscar y estudiar la manera de concretar el puesto del Trabajador Social, demostrando su rentabilidad" (Fuertes, Ros y Llopis 1985, citado por Rodríguez, 2010, p.748).

Por ello, es necesario resaltar temas propios del Trabajador Social de Empresa como son el clima social, valoración de impactos, etc., configurándose como el técnico más idóneo para acometer esta nueva filosofía de trabajar, que se pretende desde la empresa, siendo el objetivo prioritario el Bienestar Ocupacional.

La responsabilidad social empresarial (TSC)

Estas actuaciones propias del TSE nos relaciona directamente con la RSC, que es el ámbito que se ha desarrollado a partir del interés de los empresarios en mantener una relación armoniosa con la comunidad, si bien tiene bases éticas también responde a una estrategia de permanencia y legitimidad de la empresa en el mediano y largo plazo. (Garavito, 2008, p.1).

Así, la International Standard Organization (ISO) relaciona la RSE con la sostenibilidad económica, la protección del medioambiente, la salud y bienestar de una sociedad. Por ello, todas las empresas, con independencia de su tamaño, sector de actividad - localización, son responsables del impacto que provocan sus actividades y decisiones (Guía RSE, 2011).

De esta manera los grandes capitales y las empresas multinacionales tienen un importante papel que jugar, ya que pueden promover, pero también impedir o incluso perjudicar el desarrollo de políticas públicas o normativas, tanto a nivel nacional como internacional, que favorezcan un desarrollo más equilibrado y sostenible, la erradicación de la pobreza y el derecho de una vida digna. Todo ello, ha favorecido el auge del debate de la R.S.C., como herramienta para aminorar el impacto negativo de las empresas en general, y de las multinacionales en particular, sobre diferentes ámbitos: los derechos sociales, laborales, el medioambiente y en definitiva, sobre los derechos humanos. (González, 2008, p.35).

Dado el auge de la RSC y de la creciente preocupación de la empresa por el contexto social que le rodea, se abre una vía definitiva para realzar el valor del Trabajador Social en el ámbito empresarial, como instrumento garante de la sostenibilidad social de la empresa y la contribución de la misma a la mejora de la calidad de vida y el bienestar de la comunidad, que le circunda, a su vez, garantía para el desarrollo económico y mantenimiento de su actividad (Relinque y Burgos, 2008).

Por ello, el profesional del Trabajo Social se perfila como un agente idóneo para la puesta en marcha de las acciones de Responsabilidad Social de la empresa, la relación y detección de necesidades de los stakeholders (desde el enfoque de la empresa son los clientes, proveedores, trabajadores) a la promoción del cambio social, la solución de los problemas relacionales y el fortalecimiento y liberación a las personas para incrementar el Bienestar Social (FITS, 2007 citado por VII Congreso Estatal de Facultades de Trabajo Social).

\section{Hipótesis}

Una vez que se introdujo el tema que se pasó a investigar, se reflejó la hipótesis de la que parte este estudio, en la que se interpreta que la figura del Trabajador Social tiene un lugar relevante en el departamento de Recursos Humanos, en el que ejercer su labor como profesional diferenciador, dedicándose al Bienestar Ocupacional.

Objetivos

Objetivo general

- Dar importancia a la figura profesional del Trabajo Social dentro de las empresas.

\section{Objetivos específicos}

- Ampliar los conocimientos de los universitarios de Grado de Trabajo Social en el ámbito empresarial. 
Fayna Mesa Medina - El trabajo social de empresa: Otro ámbito de trabajo

- Conocer el trabajo de los profesionales que ejercen esta rama del Trabajo Social actualmente.

- Descubrir la situación actual de los Trabajadores Sociales de Empresa.

- Averiguar cómo ejercen su función en las empresas.

- Saber en qué sector empresarial se sitúan.

Justificación de la investigación

El profesional de Trabajo Social tiene como principal objetivo el Bienestar Social de los ciudadanos. En este sentido, el Trabajo Social tiene de nuevo la oportunidad de adentrarse en el ámbito empresarial, porque este tiene un papel aglutinador de los factores personales, sociales y contextuales que afectan a la productividad, tanto del trabajador como de la organización en general.

En la actualidad, se reconoce la relación entre el bienestar del trabajador y su rendimiento, el objetivo del profesional de Trabajo Social es lograr hacer mejores empresas, exitosas y socialmente comprometidas, acordes con una verdadera Responsabilidad Social Corporativa (RSC).

Este tipo de acción social de empresa tiene cabida en las grandes corporaciones, donde todavía permanece la figura del Trabajador Social. Sin embargo, el tejido empresarial español, se caracteriza por pertenecer a las Pymes que, según el Directorio Central de Empresas (DIRCE), en 2011 representaban el 99,88\% del total de empresas.

De modo que, de nuevo aquí, se abre una de las vías de emprendimiento para el Trabajo Social, mediante la prestación de servicios de atención social a las empresas, dirigidos al personal de las Pymes, de forma externalizada. Para ello, es preciso conocer las funciones profesionales en las organizaciones empresariales (Raya y Caparrós, 2010).

Es, por tanto, el momento de retomar este importante papel en las empresas y demostrar que la rentabilidad forma parte de nuestra labor.

\section{Metodología}

El objetivo general de este estudio es proporcionar visibilidad al profesional del Trabajador Social de Empresa. Además, se persigue ofrecer un mayor conocimiento de la figura del Trabajador Social de Empresa formando parte de los objetivos específicos del trabajo de investigación. La hipótesis de este estudio sitúa la figura del Trabajador Social en un lugar relevante en el departamento de Recursos Humanos, en el que este ejerce su labor como profesional diferenciador, dedicándose al Bienestar Ocupacional.

La metodología desarrollada es de corte cualitativo, de carácter descriptivo y aplicado, ya que busca la aplicación o utilización de los conocimientos adquiridos, después de implementar y sistematizar los conocimientos basados en investigación. De hecho, el uso del conocimiento y los resultados de investigación ofrecen como consecuencia un perfil riguroso, organizado y sistemático de conocer la realidad (Murillo, 2008).

Este estudio tiene un enfoque cualitativo, de nivel exploratorio, puesto que se efectúa sobre un tema poco estudiado, de carácter descriptivo y práctica o aplicada, con una sistematización rigurosa y cuidadosa en el que se busca conocer la situación actual, de manera organizada para garantizar la producción de conocimiento.

Tal y como describen los autores Taylor y Bogdan (1987), el propósito de la investigación no es solo incrementar la comprensión de la vida social por parte del investigador, sino también compartir esa comprensión con otras personas. Para empezar a presentar la realización de este apartado, comenzaremos especificando cómo se realizó la revisión bibliográfica, que se centró en una aproximación al ámbito teórico y empírico del conocimiento y visualización de los profesionales del Trabajo Social, específicamente, en el ámbito de Trabajo Social Ocupacional o de Empresa.

Por otra parte, en la investigación cualitativa existe un diálogo permanente, por lo que se 
BÚSQUEDA - Enero / Junio de 2015 - No. 14 (62 - 76)

ha elegido la entrevista como el instrumento adecuado para analizar este estudio, siendo uno de los métodos preferidos a la hora de investigar en investigación cualitativa, considerando que a este método se le denomina entrevista en profundidad.

Las entrevistas en profundidad se pueden clasificar en base a si buscan analizar muchos temas de forma general o se ciñen a un tema concreto, se podría entender que las entrevistas que tienen por objetivo el análisis de una actividad, tema o acontecimiento concreto, se la denomina entrevista enfocada. Así pues, la entrevista enfocada (Merton, Fiske y Kendall, 1956, citado en Báez y Pérez de Tudela, 2007) indaga en una experiencia concreta del informante sobre la que se desea saber y cuyos efectos quieren analizarse.

\section{Participantes}

A través del Colegio Oficial de Trabajo Social de la provincia de Valencia, se solicitó la colaboración para contactar con profesionales colegiados que ejercieran su labor en empresas privadas. Fue necesario acotar la inclusión, excluyendo a aquellos que no ejercían la profesión de Trabajo Social de Empresa en el departamento de Recursos Humanos (RRHH), siendo sólo posible incorporar a tres profesionales, por ser los únicos que cumplían el requisito mencionado. Esto se decide así, porque es el departamento de Recursos Humanos el que se encarga de atender todas las necesidades y situaciones referidas al personal humano (Mesén, 1998). Además, el Trabajador Social de Empresa realiza su labor dentro del departamento de $\mathrm{RRHH}$, dirigiendo sus acciones a humanizar las relaciones entre el capital y el trabajo, aumentando el rendimiento y el nivel de competitividad, dentro de un pensamiento de calidad de vida laboral.

\section{Técnicas e Instrumentos de recolección de} datos

La entrevista se estructura en torno a las preguntas del investigador y las respuestas del informante, lo cual produce un flujo de información que va dotando de contenidos a la entrevista. Cada tema tendrá asociada su correspondiente pregunta, con la que se redactará la entrevista. Se han escogido dieciséis preguntas, que se expondrán a cada uno de los profesionales que participan en este trabajo de investigación, mediante una entrevista enfocada. Una vez realizada la entrevista, grabada y transcrita literalmente, se analiza cada pregunta, extrayendo códigos, que en nuestro caso pasaremos a denominar conceptos claves y coincidentes, y como subcódigo conceptos diferenciales, si los hubiera, añadiendo las frases literales de los participantes del estudio.

\section{Análisis de los resultados}

Para analizar este cuadro partimos de los Temas que hemos seleccionado partiendo de la hipótesis que planteamos en el trabajo de investigación para confirmarla, o no, y también para corroborar o no, los objetivos marcados en este estudio. De cada tema le corresponde una o dos Preguntas que hemos elaborado partiendo de los temas. Los Códigos son palabras o conceptos coincidentes que tendrán en común los entrevistados, y serán "claves" para la comprensión de los Temas. Los Subcódigos serán aquellas palabras o frases, que serán destacadas por no coincidir con el resto de respuestas de las personas entrevistadas. $Y$ por último, las Frases son párrafos extraídos de las respuestas de los entrevistados que darán explicación y consistencia al Código y a los Subcódigos. A través de las coincidencias, se elaborará el resultado, estableciendo la consistencia teórico-práctica. 
Fayna Mesa Medina - El trabajo social de empresa: Otro ámbito de trabajo

Tabla 1. Relación códigos, subcódigos y memos

\begin{tabular}{|c|c|c|c|c|}
\hline TEMAS & PREGUNTAS & CÓDIGOS & SUBCÓDIGOS & FRASES \\
\hline $\begin{array}{l}\text { Descripción } \\
\text { del TSE }\end{array}$ & ¿Qué es TSE? & $\begin{array}{l}\text { Bienestar } \\
\text { Ocupacional }\end{array}$ & No hay & $\begin{array}{l}\checkmark \text { Entrevista A: "Identificación } \\
\text { de necesidades familiares y } \\
\text { socio-laborales". } \\
\checkmark \text { Entrevista B: "El trabajo } \\
\text { no es un espacio estanco, } \\
\text { hay que buscar la mejora del } \\
\text { trabajador". } \\
\checkmark \text { Entrevista C: "Todos los } \\
\text { ámbitos que rodean a un } \\
\text { puesto de trabajo: salud } \\
\text { laboral, desarrollo profesional, } \\
\text { reconocimiento y realización } \\
\text { de personas porque las } \\
\text { empresas son pequeñas } \\
\text { sociedades y trabajamos para } \\
\text { personas, y las personas son } \\
\text { las mismas en el momento } \\
\text { que salen de su casa que } \\
\text { cuando llegan al puesto } \\
\text { de trabajo; se llevan sus } \\
\text { emociones, sus problemas, y } \\
\text { la sensación de estar bien en } \\
\text { el puesto de trabajo es lo que } \\
\text { más valora un trabajador, más } \\
\text { que el sueldo". }\end{array}$ \\
\hline
\end{tabular}

Función del TSE
¿Función del TSE? Gestión de

personas

¿Ejerces otras?
Funciones de $\mathrm{RRHH}$
No hay

No hay $\checkmark$ Entrevista A: "Atención a la salud, problemas de prevención social, atención a personas con discapacidad". $\checkmark$ Entrevista B: "Entrevistas con los trabajadores, informes sociales, fomentar y trabajar la inserción al mundo laboral". $\checkmark$ Entrevista C: "Perspectiva de mejora continua, de resiliencia, de actitud positiva, de innovación".

$\checkmark$ Entrevista A: "A nivel de relaciones laborales y servicios médicos".

$\checkmark$ Entrevista B: "También me encargo de la selección de personal, con la contratación, las nóminas, los traslados".

$\checkmark$ Entrevista C: "Mediación de conflictos, asesoramiento empresa-trabajadores, diseño de proyectos junto con prevención y formación". 
BÚSQUEDA - Enero / Junio de 2015 - No. 14 (62 - 76)

\section{Departamento del TSE dentro de la empresa}

¿Colaboras en otro departamento aparte del de $\mathrm{RR} . \mathrm{HH}$ ?

¿Crees que es necesaria la figura de TSE en las empresas privadas?
Sí, labores transversales

No hay

Sí, ofrece

garantías a los trabajadores y

No hay evita conflictos.

\section{Atención al personal en fase productiva y jubi- lación}

\section{¿Trabajas con todo el personal o solo con los que acuden a ti?}

El personal jubilado ¿sigue siendo atendido?
Con todos

aquellos que

soliciten mi

colaboración

Siguen formando parte de la empresa.
No hay

En la empresa de la entrevistada B, el éxito de esta radica en no llegar a jubilarse dentro de su empresa.
¿Se atienden las necesidades familiares de los trabajadores?
Cuando afectan a Cuando la productividad solicitan. del empleado. $\checkmark$ Entrevista A: "Asesor de comisiones pluripartita, informe de peritaje social, económico, familiar y laboral".

$\checkmark$ Entrevista B: "Soy la responsable de todo lo relacionado al personal".

$\checkmark$ Entrevista C: "Departamentos con el fin de mejorar las condiciones laborales, secciones sindicales".

$\checkmark$ Entrevista A: "Digamos que está reservado para las empresas con inteligencia emocional madura y con alto nivel de compromiso social directo con sus empleados".

$\checkmark$ Entrevista B: "Somos una unidad, si nuestra vida privada y personal está totalmente relacionada con nuestra vida laboral, el trabajador rendirá mejor y más, que es lo que quiere el empresario".

$\checkmark$ Entrevista C: "Conocer cuáles son los problemas sociales de la empresa y ofrecer un plan de acción".

$\checkmark$ Entrevista A: "A demanda del cliente interno".

$\checkmark$ Entrevista B: "Todo aquel que acude a mi".

$\checkmark$ Entrevista C: "Pueden acudir los trabajadores, sus jefes, los médicos, los sindicatos".

$\checkmark$ Entrevista A: "La persona mayor y jubilados son los clientes definidos en esta empresa por una instrucción interna como uno de los objetivos de los TSE".

$\checkmark$ Entrevista B: "Es una empresa reciente, somos un centro especial de empleo y nuestra finalidad es insertar al mundo laboral ordinario a las personas".

$\checkmark$ Entrevista C: "Sí, y si no podemos intervenir derivamos a los servicios sociales correspondiente".

\section{familiares de la empresa}


Fayna Mesa Medina - El trabajo social de empresa: Otro ámbito de trabajo

$\checkmark$ Entrevista B: "Somos como una pequeña familia, tienen mi número de móvil, a veces, incluso se solucionan por vía telefónica.

$\checkmark$ Entrevista C: "Somos como una oficina base, es decir, ofrecemos información y orientación para atender las necesidades sociales mediante los recursos como guarderías públicas.

Rol del TSE

¿Puede desarrollar su especialización en toda su amplitud?
Depende de los recursos de la empresa.
Formación específica y académica del TSE
¿Crees que existe falta de conocimientos específicos en la formación inicial de TS?
Sí, en las Universidades Universidad Salamanca TSE $\checkmark$ Entrevista A: "Los ámbitos van cambiando, con las necesidades funcionales $y$ empresariales".

$\checkmark$ Entrevista B: "Se puede decir que una empresa es como la sociedad; existen situaciones de todo tipo y hay que estar muy informado para poder atender a todas las situaciones, e igual que en la sociedad a veces en las empresas no hay recursos ni respuesta".

$\checkmark$ Entrevista C: "En la empresa se trabaja con pocos recursos económico, hay que usar, lo que hay, no tenemos muchos apoyos, solo nuestra experiencia y la relación de ayuda a los trabajadores".

e $\checkmark$ Entrevista A: "Desconozco la actualidad, pero he colaborado con alguna universidad" "Yo estudié una asignatura de TSE en Salamanca en el año 1990". $\checkmark$ Entrevista B: "Cuando estudié en 1996, estudié una asignatura relacionada con $\mathrm{RRHH}$ y empresa, No supieron inculcarme la importancia de esta asignatura y es ahora cuando veo la importancia de ello".

$\checkmark$ Entrevista C: "En la Universidad se habla siempre desde la perspectiva de SS.SS, pero el TS es algo más, hay una perspectiva más; amplia que no se aplica. Hay que ampliar y ver nuevas perspectivas. Se ve esa necesidad en la Universidad pero no arranca, no termina de cuajar". 
BÚSQUEDA - Enero / Junio de 2015 - No. 14 (62 - 76)

¿Tienes formación Sólo en materias $\quad \boldsymbol{V}$ No hay continua para es- transversales. pecializarte en esta rama?

\section{RSC ejercida por TSE}

\section{Apoyo desde los Colegios de TS}

$\checkmark$ ¿RSC tiene relación con TSE? $\checkmark$ Si, EI TSE

es uno de los profesionales más apropiado para ejecutar la RSC. $\checkmark$ No hay

$\checkmark$ No utilizo recursos del ha Colegio a pesar de estar colegiada.

$\checkmark$ Entrevista A: "Hubo un tiempo en el que anualmente se programaba formación específica para TSE con altísima calidad de los cursos, ahora ya no es específica".

$\checkmark$ Entrevista B: "La formación que recibo es tanto para mí como para mis compañeros".

$\checkmark$ Entrevista C: "Aunque todo es necesario, no recibo formación sobre TSE".

$\checkmark$ Entrevista A: "En Telefónica no existe relación directa entre RSC y TSE, pero puede influir de forma transversal como, por ejemplo, como voluntariado, promoviendo el cuidado del medio ambiente o participando como mentor de Star Ups o ThingBig".

$\checkmark$ Entrevista B: "Si las empresas son responsables de su entorno, los TSE trabajamos con las personas, el entorno y la sociedad".

$\checkmark$ Entrevista C: "Somos los gestores ideales para desarrollar el rol de la empresa como responsables sociales, somos los más conocedores en el ámbito de lo social".

¿Existe apoyo $\quad \checkmark$ Normalmente, desde los Colegios Oficiales para desempeñar el perfil de TSE? apoyan cualquier demanda profesional.

\section{$\checkmark$ Entrevista A: "Siempre ha estado respaldando y apoyando cualquier consulta profesional. Es imprescindible".}

$\checkmark$ Entrevista B: "Lo estoy porque es obligatorio para mi puesto de trabajo, pero no lo necesito para nada".

$\checkmark$ Entrevista C: "Desde la creación de la nueva Junta, hay una iniciativa para fomentar el TSE". 
Fayna Mesa Medina - El trabajo social de empresa: Otro ámbito de trabajo

\section{Resultados}

En la "Descripción del TSE" todos los integrantes que formaron parte de la investigación han respondido, con el mismo significado, haciendo referencia a Bienestar Ocupacional, que significa:

El trabajo no es un espacio estanco, hay que buscar la mejora del trabajador; identificación de necesidades familiares y socio-laborales; todos los ámbitos que rodean a un puesto de trabajo: salud laboral, desarrollo profesional, reconocimiento y realización de personas, por que las empresas son pequeñas sociedades $y$ trabajamos para personas, y las personas son las mismas en el momento que salen de su casa que cuando llegan al puesto de trabajo, se llevan sus emociones, sus problemas, y la sensación de estar bien en el puesto de trabajo es lo que más valora un trabajador, más que el sueldo".

Se hace referencia a las "Funciones del TSE" mediante una relación común con "Gestión de personas" que significa:

"Atención a la salud, problemas de prevención social, atención a personas con discapacidad; entrevistas con los trabajadores, informe sociales, fomentar y trabajar la inserción al mundo laboral; perspectiva de mejora continua, de resiliencia, de actitud positiva, de innovación".

Para centrarnos en el "Rol del TSE" se destacó el código Depende de los recursos de la empresa:

"Los ámbitos van cambiando, con las necesidades funcionales y empresariales. "se puede decir que una empresa es como la sociedad; existen situaciones de todo tipo y hay que estar muy informado para poder atender a todas las situaciones, e igual que en la sociedad a veces en las empresas no hay recursos ni respuesta; en la empresa se trabaja con pocos recursos económicos, hay que usar lo que hay, no tenemos muchos apoyos, solo nuestro expertise y la relación de ayuda a los trabajadores".
El "Departamento del TSE dentro de la empresa" se relaciona con labores transversales entendiéndose:

"Asesor de comisiones pluripartita, informe de peritaje social, económico, familiar y laboral. Soy la responsable de todo lo relacionado con el personal; Departamentos con el fin de mejorar las condiciones laborales, secciones sindicales; digamos que está reservado para las empresas con inteligencia emocional madura y con alto nivel de compromiso social directo con sus empleados. Somos una unidad, si nuestra vida privada y personal está totalmente relacionada con nuestra vida laboral, el trabajador rendirá mejor y más, que es lo que quiere el empresario; conocer cuáles son los problemas sociales de la empresa y ofrecer un plan de acción".

En relación la "Atención al personal en fase productiva y jubilación", la relación es $\underline{\text { Con todos }}$ aquellos que soliciten mi colaboración porque siguen formando parte de la empresa con la siguiente explicación:

"A demanda del cliente interno; todo aquel que acude a mi. Pueden acudir los trabajadores, sus jefes, los médicos, los sindicatos; la persona mayor y jubilados son los clientes definidos en esta empresa por una instrucción interna como uno de los objetivos de los TSE; sí, y si no podemos intervenir derivamos a los servicios sociales correspondiente".

Por otra parte, tenemos el subcódigo en "La empresa de la entrevistada B. el éxito de esta radica en no llegar a jubilarse dentro de su empresa", porque "es una empresa reciente, somos un centro especial de empleo y nuestra finalidad es insertar al mundo laboral ordinario a las personas".

En el tema "Atención a los familiares de la empresa", los entrevistados coinciden con la respuesta. "Cuando afectan a la productividad del empleado".

"Situaciones de adiciones, situaciones en el ámbito familiar que requiera de nuestra 
BÚSQUEDA - Enero / Junio de 2015 - No. 14 (62 - 76)

intervención. "Somos como una oficina base, es decir, ofrecemos información y orientación para atender las necesidades sociales mediante los recursos como guarderías públicas".

Sin embargo, existe un subcódigo el cual indica que Cuando lo solicitan y explican que "Somos como una pequeña familia, todos tienen mi número de móvil y, a veces, incluso se solucionan los problemas por vía telefónica.

En la "RSC ejercida por TSE" se ha destacado que "El TSE es uno de los profesionales más apropiado para ejecutar la RSC", justificándose con las siguientes respuestas:

"En Telefónica no existe relación directa entre RSC y TSE, pero puede influir de forma transversal como, por ejemplo, voluntariado; promoviendo el cuidado del medio ambiente o participando como mentor de Star Ups o ThingBig. Pero si las empresas son responsables de su entorno, IOS TSE trabajamos con las personas, el entorno y la sociedad. Somos los gestores ideales para desarrollar el rol de la empresa como responsables sociales, somos los más conocedores en el ámbito de lo social".

Como "Formación específica y académica del TSE" con el código "Universidad" responden que:

"Cuando estudié en 1996, estudié una asignatura relacionada con $\mathrm{RRHH}$ y empresa, no supieron inculcarme la importancia de esta asignatura y es ahora cuando veo la importancia de ello. En la Universidad se habla siempre desde la perspectiva de SS.SS, pero el TS es algo más, hay una perspectiva más amplia que no se aplica. Hay que ampliar y ver nuevas perspectivas, se ve esa necesidad en la Universidad pero no arranca, no termina de cuajar".

Pero, se obtiene un subcódigo que indica que "Universidad de Salamanca TSE" explicando que "Desconozco la actualidad, pero he colaborado con alguna universidad" y estudié una asignatura de TSE en Salamanca en el año 1990.
Por último, se necesita averiguar si existe "Apoyo desde los Colegios de TS", respondiendo con el código "Normalmente apoyan cualquier demanda profesional", y afirmando que:

"Siempre ha estado respaldando y apoyando cualquier consulta profesional, es imprescindible. Y desde la creación de la nueva Junta, hay una iniciativa para fomentar el TSE".

Sin embargo, hay un subcódigo afirmando que "No utilizo los recursos del Colegio a pesar de estar colegiada" explicando: "Estoy colegiada porque es obligatorio para mi puesto de trabajo, pero no utilizo el recurso para nada".

\section{Conclusiones}

Partiendo del concepto de Bienestar Ocupacional, los profesionales pertenecientes al estudio buscan identificar las necesidades del trabajador, incluso antes de que aparezcan, informando y orientando a todos los trabajadores, sin hacer distinciones entre las diferentes categorías, aunando las distintas situaciones que pueden mejorar el rendimiento del trabajador, al tener una atención individualizada y que tiene presente todas las esferas de la vida de una persona.

Esa es la diferencia del TSE, que ve al trabajador como persona y no solo como una figura humana de rendimiento y productividad. Así, afirmamos que los TSE son los profesionales que poseen las herramientas necesarias para ocuparse del Bienestar Ocupacional, al englobar los factores personales, sociales y económicos en el ámbito laboral, sin dejar de tener presente la política de la empresa.

Sobre las funciones que ejercen en la actualidad los TSE, existe cierto vacío en algunos ámbitos de actuación; ello se debe a que se desconoce el límite profesional que existe entre las distintas profesiones que ejercen en el Departamento de Recursos Humanos, ya que este es muy amplio y variado. 
Sin embargo, las funciones que nos indican los profesionales que han participado en este trabajo son las mismas que se indican en la teoría, pero queremos hacer hincapié en una; el informe social, del que es responsable y experto el TS, en este caso específico el TSE. Con este informe se puede obtener un conocimiento holístico del trabajador, del tal forma que así sí se puede ofrecer una mayor atención al trabajador, favoreciendo un clima de bienestar y, por ende, aumentando el rendimiento de este.

Por otro lado, queremos hacer constar en este apartado que el departamento de $\mathrm{RRHH}$ es el idóneo para la figura del TSE, porque busca concretar y conservar el esfuerzo, las experiencias, la salud, los conocimientos y las habilidades; en fin, los aspectos físicos, sociales y psicológicos de los miembros de la organización, en beneficio de la persona, la institución y del país en general (Valverde, 1990).

Si hablamos del departamento de RSC consideramos que incorporando a un TSE se genera un beneficio que no ofrece otra profesión: el TSE un experto en necesidades sociales, que conoce los recursos existentes para cubrirlas, especializado en informar y orientar a las personas, independientemente de su cultura, etnia, situación o edad.

Para finalizar este apartado, creemos que es necesario que se planteen desde las Facultades de Trabajo Social y de los colegios profesionales porque, a través del programa de prácticas, las empresas conocerán qué significa y promueve la figura del profesional de TSE, comprobando los beneficios. Asimismo, desde los colegios oficiales de TS puede promocionar esta figura y ofrecer una especialización muy interesante para la sociedad en general y para los trabajadores en particular.

\section{Referencias bibliográficas}

Albacete, R., Gómez, C Ma . Meseguer, V., y Avilés, M. (2014). Responsabilidad Social Corporativa: una visión pluridisciplinar del compromiso empresarial. I Congreso Internacional de Facultades y Escuelas de Trabajo Social. Murcia.

Alvarado, $\mathrm{M}^{\mathrm{a}}$. (2007). Un nuevo campo de aplicación profesional de Trabajo Social: El Área de Recursos Humanos. El caso de Costa Rica. Recuperado de http://www. binasss.sa.cr/revistas/ts/v31n70/art4.pdf

Barranco, M., Gómez, M T., Roquero, F. y Rubí, R. (1989). El Trabajo Social de Empresa potenciador de recursos sociales. II Seminario de Trabajo Social de Empresa. Madrid.

Bernard, M. (1967). El servicio social de la empresa. Barcelona: Instituto Católico de Estudios Sociales.

Carrasco, J. (2009). Empresa y trabajo social, ¿una relación de ida y vuelta? Humanismo y trabajo social, 8, 69-83.

Castillo, A. (2010). El imaginario del trabajo social en las tesinas de fin de estudios 19381983: La evolución del Trabajo Social de empresa. Ed. Departamento de Trabajo Social y Servicios Sociales Escuela Universitaria de Trabajo Social Universidad Complutense de Madrid. (Recuperado en http://eprints.ucm.es/12263/).

DelaCuesta, M.yValor, C. (2003). Responsabilidad social de la empresa: Concepto, medición y desarrollo en España. Boletín económico de ICE, (2755) Recuperado en: http://www. revistasice.info/cachepdf/BICE_2755_0719__843B2AFA16833BD45F65BF4833 2D2587.pdf

De Miguel, L. (2011). La Responsabilidad Social Empresarial en la pequeña y mediana empresa. Guía de Buenas Prácticas. 
BÚSQUEDA - Enero / Junio de 2015 - No. 14 (62 - 76)

Ed. Ministerios de Industria, Turismo y Comercio.

ERcova (s.f.) Técnico/a en Responsabilidad Social Corporativa de la empresa. Ed: Agrupación de desarrollo Crescova.

Garavito, C. (2008). Responsabilidad Social Empresarial y mercado de trabajo. Revista Economía, Fondo Editorial de la Pontificia Universidad Católica del Perú, 61, 81-104.

González, E. (2008). La responsabilidad social corporativa: nuevo ámbito de intervención del trabajo social. Trabajo Social Hoy, 54. Ed. Colegio Oficial de Diplomados en Trabajo Social y Asistentes Sociales de Madrid.

Gorostidi, B. y Cebrián, C. (1999). Trabajo Social en pequeñas y medianas empresas.

López, E y Chaparro M.Y. (2006) Competencias laborales del trabajador social vistas desde el mercado laboral. Tabula Rasa, 5.

Marcuellos, C. (2006) Una revisión del Trabajo Social en el mundo de la empresa. Acciones e investigaciones sociales, Extra $1,457$.

Mesén, R. (1998). Trabajo Social y Recursos Humanos. Revista de Trabajo Social, 23 (53), 1052-1060. Ed. Caja Costarricense de Seguro Social Sección de Trabajo Social.

Raya, E. y Caparrós, N. (2013). Trabajo social en las relaciones laborales y la empresa: vías para el emprendimiento. Revista de trabajo y acción social, 52, 338-356. Ed. Colegio Oficial de Diplomadas y Diplomados en Trabajo Social y Asistentes Sociales de Málaga.
Relinque, F. y Burgos, E (2008) La Responsabilidad Social Corporativa. Un ámbito profesional propicio para el trabajo social. Comunicación presentada en el VII Congreso estatal de facultades de trabajo social (Recuperado de http://www10. ujaen.es/sites/default/files/users/factra/ Congreso/13.pdf

Rodríguez, A. (2006). Responsabilidad social empresarial, calidad de vida y trabajo social. Trabajadora Social, 10, 165-185. Ed. Universidad Nacional de Colombia.

Rodríguez, E. (2010). Alternativa para el Trabajador Social de Empresa. Miscelánea Camillas, 69(13), 743-760.

Roldán, J. (1990) El Trabajo Social en la empresa. Colectivo de Trabajadores Sociales de Empresa de Madrid. Documentación Social Cáritas, 79, 181-192.

Soto, J.C. (1991) El trabajo social de empresa, un campo en proceso de cambio. Análisis de algunos factores que influyeron en su evolución. Cuadernos del Trabajo Social, 4(5), 241-247. Ed. Universidad Complutense.

Valverde, L. (1990). El Trabajo Social en la Empresa. Revista Ciencias Sociales, 47, 73-80. Ed. Universidad de Costa Rica. 